\title{
Changes due to age in internodal length in the sural nerve in man
}

\author{
R. G. LASCELLES ${ }^{1}$ AND P. K. THOMAS \\ From the Institute of Neurology, Queen Square, and the Royal Free Hospital, London
}

In the peripheral nerve trunks of vertebrates, the distance between the nodes of Ranvier is normally greater on fibres of larger diameter than on those of smaller size (Ranvier, 1875; Key and Retzius, 1876). At the time of myelination during development, internodal length is uniformly short, the nodes being spaced about 150 to $300 \mu$ apart. Each internode represents the territory of an individual Schwann cell and it is of interest that the basic length of Schwann cells in tissue culture is of this order (Weiss and Wang, 1945). As growth proceeds, the number of nodes normally remains constant and the internodes therefore increase in length (Boycott, 1904). It has been shown that there is a direct proportionality between internodal length and the growth in length of the part in which the nerve lies (Vizoso, 1950; Thomas, 1955). All the fibres in a peripheral nerve trunk do not become myelinated at the same time and those that become myelinated later remain of smaller diameter (Thomas, 1956). As these fibres will be stretched less during growth, their internodal length will be shorter (Vizoso and Young, 1948; Vizoso, 1950; Thomas, 1955). The dependence of internodal length on growth is also shown by the observation that in nerve fibres that have regenerated after Wallerian degeneration in an adult animal when growth has ceased, internodal length is short on fibres of all diameters (Hiscoe, 1947; Vizoso and Young, 1948; Cragg and Thomas, 1964b).

During development, the growth rates of internodal length and fibre diameter differ and the precise relationship between these two variables in a nerve is therefore complex and alters during development (see Thomas, 1955). In adult mammalian nerves, including man, it is approximately linear (Vizoso and Young, 1948; Vizoso, 1950). However, a number of authors have drawn attention to the fact that irregularities of internodal length occur, especially in the nerves of older animals. This was shown for human nerves by Vizoso (1950), who

'Present address: Department of Neurology, Guy's Hospital, London, S.E.1. considered that abnormally short internodes for a $\frac{\overline{\bar{c}}}{\overrightarrow{\widehat{D}}}$ given diameter might result either from segmental $\stackrel{\varnothing}{\stackrel{D}{~}}$ demyelination and remyelination, or as a result of complete degeneration of fibres followed by regener- $\vec{A}$ ation. Other abnormalities also occur in the peripheral nerves in later life, including occlusive $\vec{\omega}$ vascular disease involving the vasa nervorum, $\stackrel{\circ}{S}$ connective tissue overgrowth, and loss of nerve fibres (Cottrell, 1940). The measurement of 0 internodal length has been found to be a useful means of investigating the nature of the pathologicat $\overrightarrow{.}$ process in human peripheral neuropathies (Thomas 0 and Lascelles, 1965, 1966). It therefore became neces 은 sary to determine the incidence of irregularities of internodal length in normal subjects and the resulf obtained for the sural nerve are reported here. This 음 nerve was chosen for study as it is frequently useg for diagnostic biopsy.

\section{MATERIAL AND METHODS}

The specimens of sural nerve were obtained at necropsy from patients who died at the National Hospitals for Nervous Diseases or at the Royal Free Hospital, usually within 24 hours of death. They were taken from the nerve at the level of the lateral malleolus, fixed in $4 \% \mathbb{D}$ formaldehyde in a solution of $0.9 \%$ sodium chloride and stored in this until required.

The nerves from 14 patients ranging in age from 183 to 80 years were examined, the ages, sex, and cause of

TABLE I

AGE, SEX, AND CAUSE OF DEATH OF SUBJECTS

\begin{tabular}{rrrll} 
Subject No. & Age & Sex & Cause of Death \\
\hline 1 & 18 & F & Post-necrotic hepatic cirrhosis \\
2 & 31 & M & Pulmonary embolism \\
3 & 32 & F & Pituitary tumour \\
4 & 37 & M & Cerebral glioma \\
5 & 45 & M & Pontine haemorrhage \\
6 & 48 & F & Cerebral abscess \\
7 & 50 & M & Cerebral haemorrhage \\
8 & 54 & M & Cerebral glioma \\
9 & 60 & M & Cerebral glioma \\
10 & 62 & M & Cerebellar haemorrhage \\
11 & 68 & M & Myocardial infarction \\
12 & 73 & F & Cerebral infarction \\
13 & 74 & F & Cerebral haemorrhage \\
14 & 80 & F & Bronchopneumonia &
\end{tabular}


death being given in Table 1. No patient showed clinical evidence of peripheral neuropathy.

For the examination of internodal length, after washing in distilled water, the nerve was fixed for 12 to 24 hours in $1 \%$ osmium tetroxide, washed again, and then macerated for one to two days in a mixture of two parts of glycerol to one of water. Single fibres were teased out in pure glycerol with a dissecting microscope using fine mounted needles and transferred to a slide on which a drop of creosote had been placed. After approximately 20 myelinated fibres had been accumulated, covering the whole range of fibre diameters, the fibres were arranged in parallel array, the creosote drained off and the slide blotted with filter paper. The fibres were then mounted in Canada balsam. Measurements of internodal length were made at a magnification of 100 and of fibre diameter at 1,000. Five measurements of diameter were made along each internode, the mean of these readings being taken. Further details of the technique, together with an assessment of the errors involved, are given by Vizoso and Young (1948) and by Thomas and Young (1949).

\section{RESULTS}

In the subjects under the age of 65 , the correlation between internodal length and diameter was found to be close, especially in the younger subjects. The length of individual internodes has been plotted against their diameter for the 18-, 45-, and 62-yearold subjects in Figs. 1-3, and these results are representative of those obtained from the other subjects in this age range. Internodal length varies from a value of $\sim 0.15 \mathrm{~mm}$., corresponding to a fibre diameter of $2 \mu$, to $1-1.2 \mathrm{~mm}$. at a diameter of $12 \mu$, with occasional internodes as long as $1 \cdot 3$ $\mathrm{mm}$. The relationship is approximately linear and assuming $y=a+b x$, where y represents internodal length in millimetres and $x$ fibre diameter in microns, regression lines have been calculated by the method of least squares. The regression coefficients for all the nerves examined are shown in Table II. The

\section{TABLE II}

NUMBER OF INTERNODES EXAMINED (n), Y-AXIS INTERCEPTS (a), REGRESSION COEFFICIENTS (b), AND RESIDUAL VARIANCE ABOUT REGRESSION LINES $\left(\sigma_{r}{ }^{2}\right)$

\begin{tabular}{rrrrr} 
Subject No. & $\boldsymbol{n}$ & \multicolumn{1}{c}{$\boldsymbol{a}$} & $\boldsymbol{b}$ & $\sigma_{\boldsymbol{r}}{ }^{2}$ \\
\hline 1 & 90 & -0.020 & 0.100 & 0.0019 \\
2 & 69 & -0.033 & 0.094 & 0.0061 \\
3 & 65 & -0.092 & 0.103 & 0.0056 \\
4 & 77 & -0.018 & 0.097 & 0.0038 \\
5 & 87 & 0.052 & 0.074 & 0.0081 \\
6 & 126 & 0.031 & 0.076 & 0.0069 \\
7 & 57 & 0.012 & 0.072 & 0.0019 \\
8 & 92 & 0.009 & 0.090 & 0.0050 \\
9 & 143 & 0.014 & 0.078 & 0.0040 \\
10 & 80 & -0.070 & 0.082 & 0.0053 \\
11 & 89 & 0.035 & 0.073 & 0.0094 \\
12 & 88 & -0.002 & 0.060 & 0.0154 \\
13 & 138 & -0.034 & 0.067 & 0.0085 \\
14 & 101 & -0.034 & 0.079 & 0.0313
\end{tabular}
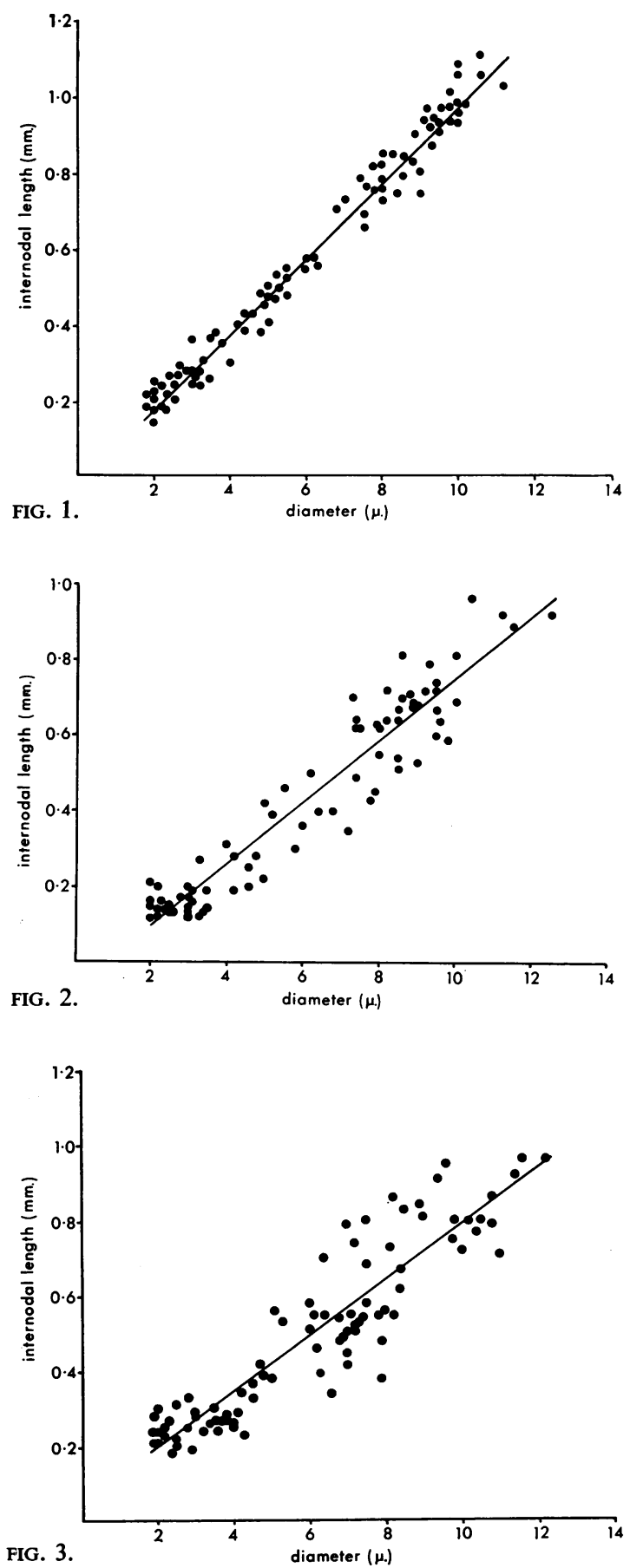

FIGS. 1, 2, and 3. Relationship between internodal length and fibre diameter for 18-year-old subject (Fig. 1); for 45-year-old subject (Fig. 2); for 62-year-old subject (Fig.3). Individual internodes with calculated regression line. 
residual variances about the regression lines $\left(\sigma_{\mathrm{r}}^{2}\right)$ are also given.

For the subjects over the age of 65 , the scatter of the observations is considerably greater. The plot of the length of individual internodes against their diameter for the two oldest subjects, aged 74 and 80 , is shown in Figures 4 and 6. Linear regression lines have also been fitted. For the four subjects over the age of 65 , the mean value of $b$ is 0.070 , as compared with 0.087 for the nerves from the 10 subjects below the age of 65 . The mean regression line for the subjects under the age of 65 is shown by the interrupted line in Figures 4 and 6. The reduced slope in the

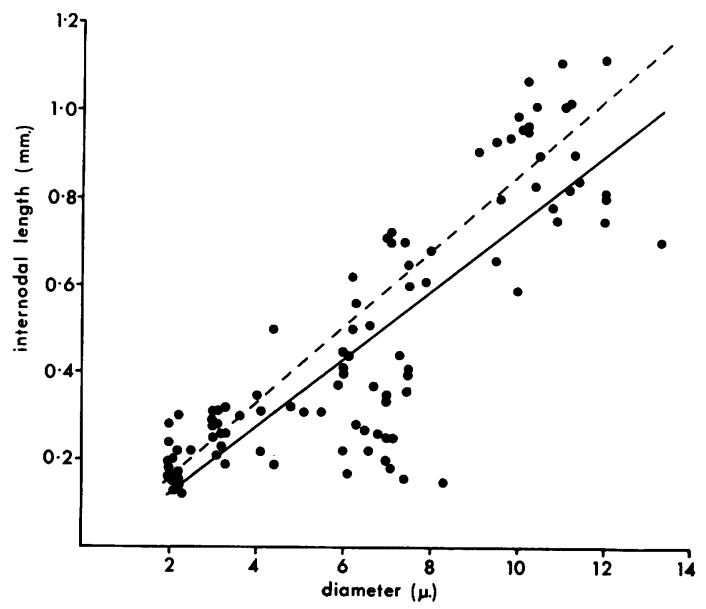

FIG. 4. Relationship between internodal length and fibre diameter for 74-year-old subject. Calculated regression line (continuous line). The interrupted line is the mean regression line for the subjects under the age of 65 .

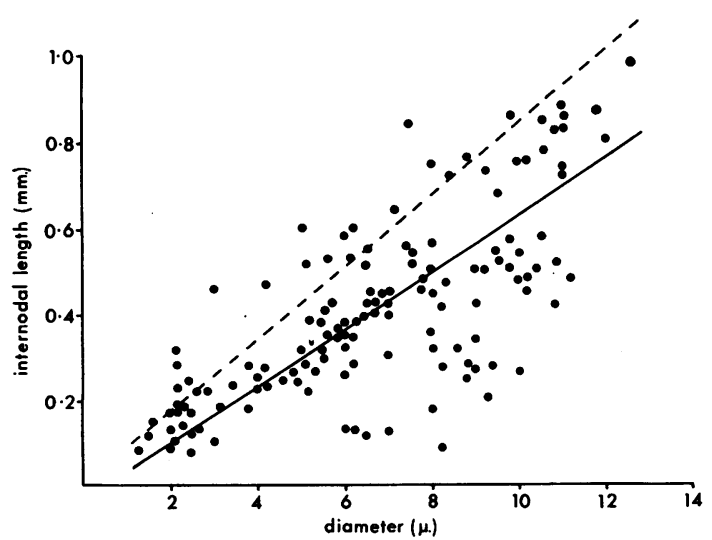

FIG. 6. Relationship between internodal length and fibre diameter for 80-year-old subject. Calculated regression line (continuous line). The interrupted line is the mean regression line for the subjects under the age of 65 . nerves from the older subjects is explained by the presence of internodes of shorter length than normals mainly in the medium and small diameter range.

In Figs. 5 and 7, the values for the 74- and 80 -year $=$ old subjects have been plotted in the way suggested by Fullerton, Gilliatt, Lascelles, and Morgan Hughes (1965). The lengths of the internodes on each? fibre have been plotted against the diameter of the widest internode and the points joined by a verticak. line. The points on these lines therefore represent the distribution of internodal lengths on single fibres. From Figs. 5 and 7 it will be seen that someof the abnormally short internodes are on fibres that

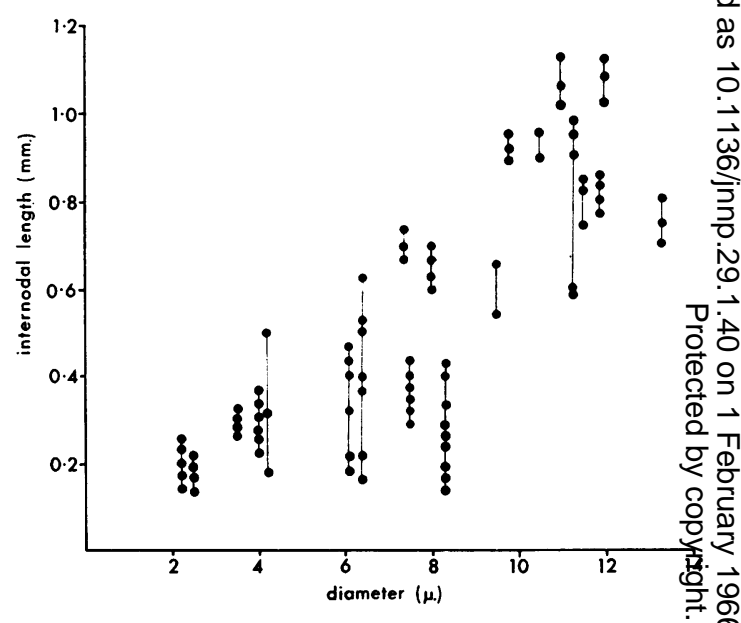

FIG. 5. Observations from Fig. 4 with internodal lengths. from individual fibres plotted against diameter of widest internodal segment and joined by vertical line.

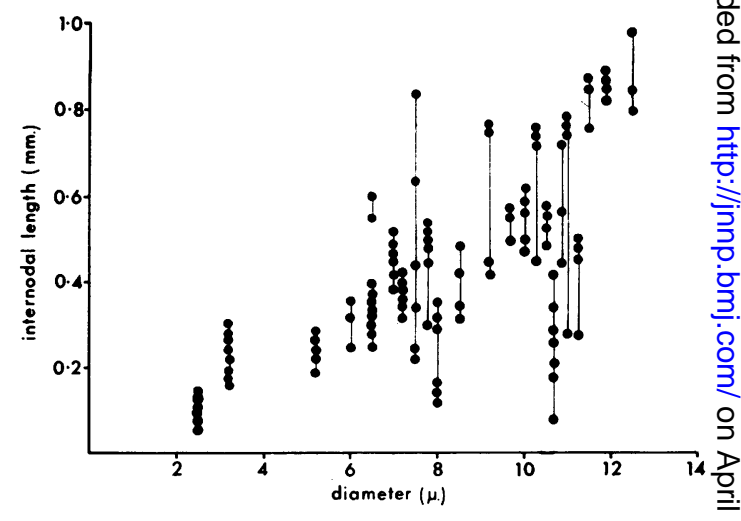

FIG. 7. Observations from Fig. 6 with internodal lengths from individual fibres plotted against diameter of widest $N$ internodal segment and joined by vertical line. 
also possess internodes of normal length, whereas on others, the internodes are of uniformly short length. In the former, the shorter internodes were usually of smaller diameter than the normal internodes in the same fibre. From the results of Fullerton et al. (1965) and Thomas and Lascelles (1965), it is known that fibres that have regenerated after Wallerian degeneration show uniformly short internodes, whereas those that have undergone segmental demyelination and remyelination show internodes of both normal and reduced length. It is interesting that both types of fibre are seen in the present material.

Abnormally long internodes were not encountered. In the subjects over the age of 65 , where the other irregularities of internodal length were observed, the longer internodes for each diameter were distributed around the mean regression line for the younger subjects (see Figs. 4 and 6).

\section{DISCUSSION}

In assessing the significance of the present results, the question of sampling has to be considered. In obtaining the fibres for measurement, no attempt was made to select either normal or abnormal fibres. The procedure employed was to take the first 20 fibres, covering the whole range of fibre sizes, that were most readily isolated. It is of interest that the results are closely comparable to those obtained by Vizoso (1950) in the ulnar and anterior tibial nerves from three subjects spanning the same age range and derived from the measurement of a considerably greater number of internodes in each nerve.

The present observations indicate that irregularities of internodal length are uncommon in the sural nerve of subjects under the age of 65 . They are frequently encountered in the older age groups and, as was observed by Vizoso (1950), appear to be the result both of segmental demyelination and remyelination, and regeneration after complete degeneration of the fibres. Segmental demyelination and remyelination was the more common explanation. This process was originally noticed in the nerves from healthy animals by Renaut (1881), who thought that the short intercalated segments were part of the normal growth process. They were observed, especially in the nerves of older animals, by subsequent workers and interpreted as a degenerative process (e.g., Thomas and Young, 1949). The phenomenon was studied in detail by Lubińska (1958).

Vizoso (1950) observed some fibres in elderly subjects with internodes that were of uniform length but inappropriately long for the diameter of the fibre. It was considered that the diameter had decreased without degeneration of the fibre. Vizoso also found internodes in excess of $1.2 \mathrm{~mm}$. in length that were longer than any he had encountered in the younger subjects. It was suggested that when local degeneration of the myelin sheath occurs, an adjacent intact Schwann cell may extend its territory. No clear indication of either of these alterations was seen in the present results.

Various investigators have reported mild slowing of nerve conduction in later life (e.g., Wagman and Lesse, 1952; Norris, Shock, and Wagman, 1953) and the histological abnormalities we have found are likely to be relevant in this connexion. Norris et al. reported that in the ulnar nerve the slowing began after the age of 30 , but in a personal series on the median nerve in the forearm (Thomas, unpublished), significant slowing was not observed until after the age of 60 . However, the situation may well vary between different nerves, depending upon whether or not the nerve is exposed to recurrent external pressure or passes through a region where an 'entrapment neuropathy' (Kopell and Thompson, 1963) is likely to occur. The changes that Vizoso (1950) observed in internodal length in later life were considerably less evident in the facial than in the ulnar and lateral popliteal nerves. Conduction velocity is slowed by conditions producing segmental demyelination (Kaeser, 1962; McDonald, 1963; Cragg and Thomas, 1964a) and probably never recovers completely (Cragg and Thomas, unpublished results). Similarly, conduction velocity is reduced in fibres regenerating after Wallerian degeneration (Berry, Grundfest, and Hinsey, 1944; Sanders and Whitteridge, 1946) and may never return to normal (Cragg and Thomas, 1964b).

Depression or loss of the ankle jerks is a common finding in the elderly (Critchley, 1931; Howell, 1949), as is impaired appreciation of vibration in the feet (Pearson, 1928). The anatomical localization of the lesions accounting for these changes has never been satisfactorily established, but the present results suggest that the alterations observed in the peripheral nerves may be responsible. The unequal involvement of fibres will lead to temporal dispersion of conduction. This, as in demyelinating peripheral neuropathies such as that due to diabetes (Thomas and Lascelles, 1965, 1966), is likely to result in loss of tendon reflexes and vibration sense since they both depend upon the ability of the nerve to conduct a synchronous volley of impulses.

The cause of the degenerative changes occurring in peripheral nerve fibres in later life is unknown. Ischaemia secondary to occlusive disease of the vasa nervorum is a possible explanation and studies 
on internodal length in the peripheral nerves of patients with occlusive vascular disease in the legs, particularly in the younger age groups, would be of interest. The cumulative effects of repeated minor trauma throughout life may also be important.

\section{SUMMARY}

Measurements of internodal length and diameter were made on isolated nerve fibres, after staining with osmium tetroxide, from the sural nerves of subjects aged between 18 and 80 years. In the nerves from the subjects under the age of 65 , internodal length was closely correlated with fibre diameter, the relationship being approximately linear. Over the age of 65 , irregularities of internodal length were common and appeared to be the result both of segmental demyelination and remyelination, and of regeneration after complete degeneration of nerve fibres.

The significance of these findings in relation to the slowing of nerve conduction found in later life and to the loss of ankle jerks and vibration sense in the legs in the elderly is discussed.

We wish to thank Dr. R. O. Barnard, Professor W. Blackwood, Dr. E. W. Hall, and Dr. W. G. P. Mair for providing the sural nerve specimens, and Professor R. W. Gilliatt for helpful discussion. We also wish to thank Miss Jean Slatford for her help in making the statistical calculations, and Mr. W. F. Hinkes and Miss C. Botwright for technical assistance. A personal grant to one of us (P.K.T.) from the Polio Research Fund is gratefully acknowledged.

\section{REFERENCES}

Berry, C. M., Grundfest, H., and Hinsey, J. C. (1944). The electrical activity of regenerating nerves in the cat. J. Neurophysiol., 7 , 103-115.

Boycott, A. E. (1904). On the number of nodes of Ranvier in different stages of the growth of nerve fibres in the frog. J. Physiol. (Lond.), 30, 370-380.

Cottrell, L. (1940). Histologic variations with age in apparently normal peripheral nerve trunks. Arch. Neurol. Psychiat. (Chic.), 43, $1138 \cdot 1150$.
Cragg, B. G., and Thomas, P. K. (1964a). Changes in nerve conductioz in experimental allergic neuritis. J. Neurol. Neurosurg. Psychiat 27, 106-115.

- 1 (1964b). The conduction velocity of regenerated peripherâ nerve fibres. J. Physiol. (Lond.), 171, 164-175.

Critchley, M. (1931). The neurology of old age. II. Clinical manifestē tions in old age. Lancet, 1, 1221-1230.

Fullerton, P. M., Gilliatt, R. W., Lascelles, R. G., and Morgano Hughes, J. A. (1965). The relation between fibre diameter an internodal length in chronic neuropathy. J. Physiol. (Lond. 178, 26-28P.

Hiscoe, H. B. (1947). Distribution of nodes and incisures in normat and regenerated nerve fibers. Anat. Rec., 99, 447-475.

Howell, T. H. (1949). Senile deterioration of the central nervou system. Brit. med. J., 1, 56-58.

Kaeser, H. E. (1962). Funktionsprüfungen peripherer Nerven experimentellen Polyneuritiden und bei der WallerscheF Degeneration. Disch. $Z$. Nervenheilk., 183, 268-304.

Key, A., and Retzius, G. (1876). Studien in der Anatomie des Nerverp systems und des Bindegewebes. Samson and Wallin, Stockholm

Kopell, H. P., and Thompson, W. A. L. (1963). Peripheral Entrap ment Neuropathies. Williams and Wilkins, Baltimore.

Lubińska, L. (1958). Short internodes intercalated in nerve fibres. Acta Biol. exp. (Warszawa), 18, 117-136.

McDonald, W. I. (1963). The effects of experimental demyelination in peripheral nerve: a histological and electrophysiological study. II. Electrophysiological observations. Brain, 86, 501-524

Norris, A. H., Shock, N. W., and Wagman, I. H. (1953). Age changes in the maximum conduction velocity of motor fibers of huma $\bar{P}$ ulnar nerves. J. appl. Physiol., 5, 589-593.

Pearson, G. H. J. (1928). Effect of age on vibratory sensibility Arch. Neurol. Psychiat. (Chic.), 20, 482-496.

Ranvier, L. (1875). Traité Technique d'Histologie. Savy, Paris.

Renaut, J. (1881). Recherches sur quelques points particuliers-di l'histologie des nerfs. I. La gaine lamelleuse et le systen hyalin intravaginal. Arch. Physiol. norm. path., 2 ser., (Paifs)옹 $8,161-190$.

Sanders, F. K., and Whitteridge, D. (1946). Conduction velocity myelin thickness in regenerating nerve fibres. $J$. Physifj (Lond.), 105, 152-174.

Thomas, P. K. (1955). Growth changes in the myelin sheath of pere pheral nerve fibres in fishes. Proc. roy. Soc. B, 143, 380-3910

- (1956). Growth changes in the diameter of peripheral nerve fibres in fishes. J. Anat. (Lond.), 90, 5-14.

-, and Lascelles, R. G. (1965). Schwann-cell abnormalities-in diabetic neuropathy. Lancet, 1, 1355-1357. (1966). The pathology of diabetic neuropathy. To be published.

- , and Young, J. Z. (1949). Internode lengths in the nerves oqv fishes. J. Anat. (Lond.), 83, 336-350.

Vizoso, A. D. (1950). The relationship between internodal length an growth in human nerves. Ibid., 84, 342-353.

, and Young, J. Z. (1948). Internode length and fibre diameter if developing and regenerating nerves. Ibid., 82, 110-134.

Wagman, I. H., and Lesse, H. (1952). Maximum conduction velocitie of motor fibers of ulnar nerve in human subjects of various ages and sizes. J. Neurophysiol., 15, 235-244.

Weiss, P., and Wang, H. (1945). Transformation of adult Schwanm cells into macrophages. Proc. Soc. exp. Biol. (N.Y.), 58 273-275. 